\title{
Accelerated IE-GSTC Solver for Large-Scale Metasurface Field Scattering Problems using Fast Multipole Method (FMM)
}

\author{
Jordan Dugan Student Member, IEEE, Tom. J. Smy \\ and Shulabh Gupta Senior Member, IEEE
}

\begin{abstract}
An accelerated Integral Equations (IE) field solver for determining scattered fields from electrically large electromagnetic metasurfaces utilizing Fast Multipole Method (FMM) is proposed and demonstrated in $2 \mathrm{D}$. In the proposed method, practical general metasurfaces are expressed using an equivalent zero thickness sheet model described using surface susceptibilities, and where the total fields around it satisfy the Generalized Sheet Transition Conditions (GSTCs). While the standard IEGSTC offers fast field computation compared to other numerical methods, it is still computationally demanding when solving electrically large problems, with a large number of unknowns. Here we accelerate the IE-GSTC method using the FMM technique by dividing the current elements on the metasurface into near- and far-groups, where either the rigorous or approximated Green's function is used, respectively, to reduce the computation time without losing solution accuracy. Using numerical examples, the speed improvement of the FMM IE-GSTC method $\left\{O\left(N^{3 / 2}\right)\right\}$ over the standard IE-GSTC $\left\{O\left(N^{3}\right)\right\}$ method is confirmed. Finally, the usefulness of the FMM IE-GSTC is demonstrated by applying it to solve electromagnetic propagation inside an electrically large radio environment with strategically placed metasurfaces to improve signal coverage in blind areas, where a standard IE-GSTC solver would require prohibitively large computational resources and long simulation times.
\end{abstract}

Index Terms-Electromagnetic Metasurfaces, Boundary Element Methods (BEM), Electromagnetic Propagation, Generalized Sheet Transition Conditions (GSTCs), Surface Susceptibility Tensors, Fast Multipole Method, Radio Environment Engineering.

\section{INTRODUCTION}

Electromagnetic metasurfaces are 2D arrays of subwavelength resonators, which are engineered at the microscopic scale to achieve a desired macroscopic field response. By engineering the shapes, sizes and structures of these resonators forming the unit cells, metasurfaces can enable variety of wave transformations when excited with a specified input field, in reflection, transmission or both. Consequently, they have found a myriad of applications throughout the electromagnetic spectrum, ranging from radio waves to optical frequencies [1]-[7].

While the metasurfaces are constructed using subwavelength resonating particles, they themselves are electrically and physically large enabling a significant interaction with the impinging electromagnetic waves. Optical metasurfaces typically consists of millions of resonators (plasmonic or dielectric based), which are engineered at the nanoscale. On

J. Dugan, Tom J. Smy, and Shulabh Gupta are with Carleton University, Ottawa, Canada (e-mail: jordan.dugan@ carleton.ca). the other end of the spectrum, at the radio frequencies (RF), due to standard and mature printed circuit board fabrication processes, unit cells of the order of $\approx \lambda / 10$ are quite common, and the metasurfaces are several wavelengths long in typical proof-of-concept demonstrations.

However, several practical system level applications require large area metasurface sizes beyond laboratory scale experiments. Two examples are that of creating electromagnetic illusions and camouflage [8], [9], and using metasurfaces to engineer the wireless environment, for instance [8], [10]-[12] In both cases, the metasurfaces are operated in larger environments with variety of scattering objects of different shapes and sizes present. The full-wave field analysis thus becomes a computationally challenging task, with multi-scale features ranging from sub-wavelength resonators of the metasurface to large physical objects placed around them.

To make the computational problem more tractable, its accuracy maybe sacrificed as a trade-off, as is commonly done in high-frequency wave-propagations regimes where raytracing [13]-[16] and paraxial approximation based methods [17] become more viable. Alternatively, to reduce the large number of computational unknowns and avoid dense structure meshing, physical metasurface structures can be represented by an equivalent zero thickness sheet models, where they are treated as spatial discontinuities. The metasurface field interaction is governed by the so-called Generalized Sheet Transition Conditions (GSTCs), in conjunction with the spatially-varying electric and magnetic surface susceptibility, $\overline{\bar{\chi}}(\mathbf{r}, \omega)$ models of the metasurfaces [18]-[20].

While variety of numerical methods have been proposed to implement GSTCs into standard volumetric Maxwell's equation solvers; including finite difference, finite element and spectral domain methods, for instance [21]-[26]; they are still not suitable for electrically large simulations. On the other hand, Integral Equation (IE) based methods are particularly attractive as large simulation domains can be handled, since no volumetric meshing is used and only surfaces are discretized $[27]-[29]$. Consequently, zero thickness sheet models implemented using GSTCs in IE solvers (IE-GTSC) have been shown to very efficiently reproduce the macroscopic field behavior of practical metasurfaces with large number of unit cells, when compared to standard brute-force full-wave computer simulations [8], [9], [30]-[32].

While IE based numerical solvers are efficient compared to other techniques, they still require large computational 
resources as simulation domains become complex, increase in size and have more scattering objects, as is typically expected in applications involving wave propagation in large-scale radio frequency environments, for instance. To accelerate the IE based solvers further, one attractive and well-known technique is to use the Fast Multipole Method (FMM) [33]-[35]. FMM divides surface elements into localized groups and optimizes the far-group interactions to speed up the entire simulation, without losing accuracy.

In this work, in the context of solving large-scale electromagnetic problems, the IE-GSTC based solver is coupled with the FMM to accelerate the simulation speed and reduce the computational resource requirements. Specially, a 2D FMM implemented via pulse expansion functions and point matching that can handle arbitrary polarization of electric and magnetic fields/currents is proposed here, that can solve large scale GSTC/metasurface problems. While an accelerated IE solver has also been applied to large scale environments before in the literature using a volume integral equation method [36][38], the use of volume integral makes this method unable to incorporate GSTCs. Although, the FMM is commonly applied to two dimensional scattering problems [33], [35], much of what is reported in the literature is developed for either $\mathrm{TM}_{z}$ or $\mathrm{TE}_{z}$ polarization (in terms of EFIE, MFIE or CFIE integrals), which are not easily extendable to general metasurface boundaries where both the electric and magnetic fields are tightly coupled through the surface susceptibilities in the GSTCs. Here, we fill this gap, by combining FMM with GSTCs to describe a general electromagnetic boundary.

The paper is structured as follows. Sec II starts by building an application case where the concept of using metasurfaces for engineering a wireless channel is used to motivate the requirement of an accelerated IE-GSTC solver. A brief summary of IE-GSTC method is next described. Sec III describes the FMM formulation and develops it further to be integrated with the GSTCs. Sec. IV presents the numerical results by taking an example of a finite sized uniform surface and compares its performance with standard IE-GSTC implemented using Boundary Element Method (BEM). It is followed by simulating large room radio frequency environment to illustrate how metasurfaces can be used to increase signal coverage, which otherwise cannot be simulated using standard BEM solver.

\section{STANDARD IE-GSTCS}

\section{A. Metasurface Application Case}

Let us consider an illustrative example of wireless communication inside a closed space, as shown in Fig. 11(a). The space consists of doors, windows and variety of physical objects, while an access point is assumed outside the rooms, aiming to provide signal coverage inside.

At microwave frequencies $(\approx<10 \mathrm{GHz})$, the electromagnetic (EM) wave interaction with the environment is highly diffractive, due to smaller antennas, compared to the physical environment and its operating wavelengths. EM waves bounce off physical objects in the wireless environment and propagate in uncontrolled directions, creating multiple paths and signal copies at the intended receivers. This provides rich channel diversity, which is used to recover the useful signal buried inside

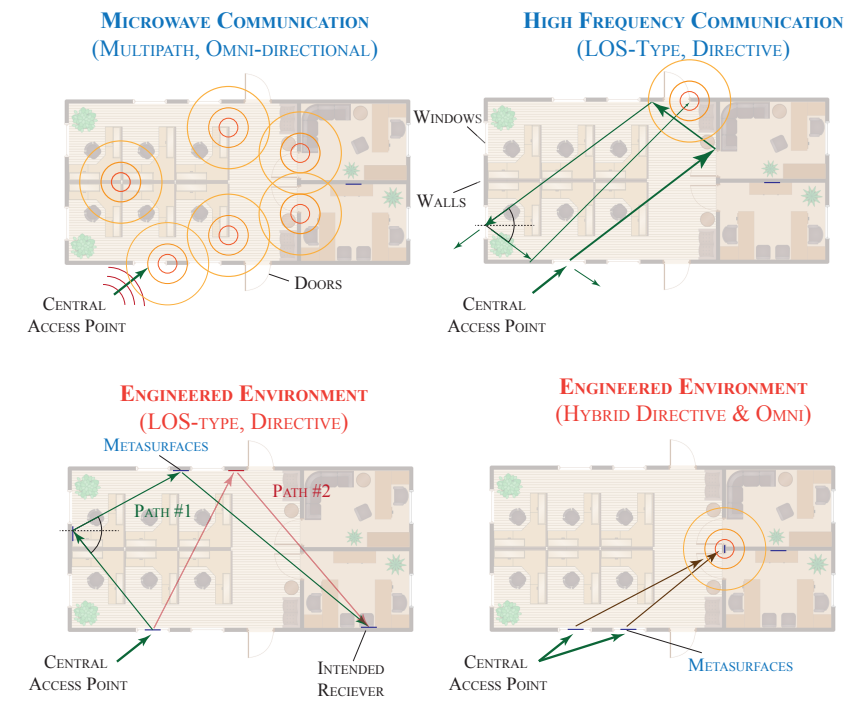

Fig. 1. The concept of strategic installation of engineered metasurfaces to guide electromagnetic waves across a complex environment, bypassing obstacles and optimizing corner diffraction for better signal coverage and improved communication performances.

the noisy non-LOS wireless channel. This is extensively used in typical Multiple Input Multiple Output (MIMO) systems to enhance the quality of signal transmission across devices [39]. As sources become more directive (typically due to increasing the frequency), waves propagate along prescribed directions, specularly reflect from walls while satisfying Snell's laws, and produce corner diffraction in the presence of sharp edges.

This directive wave propagation characteristic presents a unique opportunity to investigate a paradigm-shifting approach to engineer and design the RF environment itself, and route the electromagnetic energy to form the desired and distinct wireless signal paths. Specifically, the RF environment can be modified by introducing controlled local reflection/diffraction engineered EM objects in strategically chosen locations, instead of relying on passive reflection, and transmission through physical objects. This requirement of controlled reflection/diffraction is possible using engineered metasurfaces with specified wave transformations to improve signal coverage and throughput performance.

Consider an example scenario of Fig. 11(c) for directive communication, where the objective is to obtain the best possible signal at the intended receiver location from the source access point. With a conventional approach that utilizes the passive reflections off various walls and physical objects, such as in Fig. 1 b), receiving a weak or possibly null signal at the receiver is likely. However, if a network of smart metasurfaces are installed at strategic locations, the directive signal energy can be channelled to form a series of LOS signal paths between the access point and the receiver, as indicated by paths \#1 and \#2 in Fig. 11(c). Unlike the passive walls and reflectors of Fig. 1 $1(a-b)$, engineered metasurfaces control the output reflection and refraction angles using Generalized Snell's Laws, which makes it possible to steer beams and avoid physical obstructions and undesired corner diffractions. 
In principle, several signal paths can be devised between the source and receiver, from which the best path providing the best signal communication performance can eventually be chosen. In other situations, these metasurfaces can also be used to intentionally transform incoming directive radiation into omni-directional wavefronts, as shown in Fig. 11(d). This opens two new capabilities. 1) Metasurfaces can channel EM energy in a hybrid directive and omni-directional manner, enabling uniform illumination of selective local regions in the environment. 2) They can locally enhance the channel diversity at mm-Wave frequencies, suitable for MIMO-type systems, which is otherwise generally not available at $\mathrm{mm}$-Wave bands.

\section{B. Standard IE-GSTC Formulation}

The problem of modeling electromagnetic wave propagation in wireless radio environments described in Fig. 1. is naturally an electrically large problem which is aggravated by the presence of various metasurfaces making it a multi-scale simulation problem. In order to simplify the computational setup, without losing accuracy, physical metasurface structures can be represented using analytical models. Given that all practical metasurfaces are electrically thin, i.e. $\delta \ll \lambda_{0}$ and with sub-wavelength periodicities, $\Lambda \ll \lambda_{0}$, they can be represented using a zero thickness sheet model, that captures their effective dipolar electric and magnetic surface polarization responses. The complete electromagnetic properties of the subwavelength resonators can thus be described using compact surface susceptibility tensors $\overline{\bar{\chi}}(\mathbf{r}, \omega)$.

The electromagnetic fields around the equivalent zero thickness sheet model is then governed by the Generalized Sheet Transition Conditions (GSTCS), which are given by

$$
\begin{aligned}
& \hat{\mathbf{n}} \times \Delta \mathbf{E}=-j \omega \mu_{0} \mathbf{M}_{\|}-\hat{\mathbf{n}} \times \nabla_{\|}\left(\frac{\mathbf{P}_{n}}{\epsilon_{0}}\right) \\
& \hat{\mathbf{n}} \times \Delta \mathbf{H}=j \omega \mathbf{P}_{\|}-\hat{\mathbf{n}} \times \nabla_{\|} \mathbf{M}_{n},
\end{aligned}
$$

relating the field difference $(\Delta\{\cdot\})$ across the metasurface with the average E- and $\mathrm{H}$-fields in terms of its tangential $\{\cdot\}_{\|}$and normal surface polarizations $\{\cdot\}_{n}$, respectively, i.e.

$$
\begin{aligned}
\mathbf{P} & =\epsilon \overline{\bar{\chi}}_{\mathrm{ee}} \mathbf{E}_{\mathrm{av}}+\overline{\bar{\chi}}_{\mathrm{em}} \sqrt{\mu \epsilon} \mathbf{H}_{\mathrm{av}} \\
\mathbf{M} & =\mu \overline{\bar{\chi}}_{\mathrm{mm}} \mathbf{H}_{\mathrm{av}}+\overline{\bar{\chi}}_{m e} \sqrt{\epsilon / \mu} \mathbf{E}_{\mathrm{av}} .
\end{aligned}
$$

If for simplicity we will limit the analysis to tangential terms the GSTCs can thus be expressed as:

$$
\begin{aligned}
& \hat{\mathbf{n}} \times \Delta \mathbf{E}=-j \omega \sqrt{\mu \epsilon} \overline{\bar{\chi}}_{\mathrm{me}} \mathbf{E}_{\mathrm{av}}-j \omega \mu \overline{\bar{\chi}}_{\mathrm{mm}} \mathbf{H}_{\mathrm{av}} \\
& \hat{\mathbf{n}} \times \Delta \mathbf{H}=j \omega \epsilon \overline{\bar{\chi}}_{\mathrm{ee}} \mathbf{E}_{\mathrm{av}}+j \omega \sqrt{\mu \epsilon} \overline{\bar{\chi}}_{\mathrm{em}} \mathbf{H}_{\mathrm{av}} .
\end{aligned}
$$

Each of the surface susceptibilities, $\overline{\bar{\chi}}_{\alpha \beta}$ with $\alpha \beta \in$ $\{$ ee, $\mathrm{mm}$, em, me $\}$, is a $3 \times 3$ tensor with complex scalar elements, in general.

Now, a more general statement of the problem of interest can be formulated, as also illustrated in Fig. 2(a) consisting of a metasurface surrounded by various objects of different shapes, sizes and material. We wish to model scattering of an electromagnetic field off one or more surfaces (media interfaces, metasurfaces, object surfaces etc.) - including the coupling between surfaces. Solving the scattering problem is essentially the need to find a self-consistent solution of the excitation, the propagation of the fields and the interface conditions (e.g. GSTCs for metasurfaces) present for surfaces within the region of interest. If the surfaces present are uncharged, then these interface conditions relate the tangential components of the $\mathbf{E}$ and $\mathbf{H}$ fields across the interface or the surface. An appropriate formulation of Maxwell's equations to determine the field propagation is an Integral Equation (IE) approach.

The EM fields radiated into free-space from given electric and magnetic current sources, $\{\mathbf{J}, \mathbf{K}\}$, can be generally expressed using an IE formulation as [27], [28]:

$$
\begin{aligned}
\mathbf{E}^{\mathrm{s}}(\mathbf{r}) & =-j \omega \mu(\mathcal{L} \mathbf{J})\left(\mathbf{r}, \mathbf{r}^{\prime}\right)-(\mathcal{R} \mathbf{K})\left(\mathbf{r}, \mathbf{r}^{\prime}\right) \\
\mathbf{H}^{\mathrm{s}}(\mathbf{r}) & =-j \omega \epsilon(\mathcal{L} \mathbf{K})\left(\mathbf{r}, \mathbf{r}^{\prime}\right)+(\mathcal{R} \mathbf{J})\left(\mathbf{r}, \mathbf{r}^{\prime}\right),
\end{aligned}
$$

with $\mathbf{r}$ being the point of interest, $\mathbf{r}^{\prime}$ the position of the source current; and $\mathbf{E}^{\mathrm{s}}$ and $\mathbf{H}^{\mathrm{s}}$ the radiated (scattered) fields from the surface ${ }^{1}$ The field operators are given by:

$$
\begin{aligned}
(\mathcal{L} \mathbf{C})\left(\mathbf{r}, \mathbf{r}^{\prime}\right) & =\int_{\ell}\left[1+\frac{1}{k^{2}} \nabla \nabla \cdot\right]\left[G\left(\mathbf{r}, \mathbf{r}^{\prime}\right) \mathbf{C}\left(\mathbf{r}^{\prime}\right)\right] d \mathbf{r}^{\prime} \\
(\mathcal{R} \mathbf{C})\left(\mathbf{r}, \mathbf{r}^{\prime}\right) & =\int_{\ell} \nabla \times\left[G\left(\mathbf{r}, \mathbf{r}^{\prime}\right) \mathbf{C}\left(\mathbf{r}^{\prime}\right)\right] d \mathbf{r}^{\prime}
\end{aligned}
$$

with $\mathbf{C} \in\{\mathbf{J}, \mathbf{K}\} . G\left(\mathbf{r}, \mathbf{r}^{\prime}\right)$ represents the Green's function which, for a $2 \mathrm{D}$ case, is given by the Hankel function of the $2^{\text {nd }}$ kind,

$$
G\left(\mathbf{r}, \mathbf{r}^{\prime}\right)=H_{0}^{(2)}\left(\mathbf{r}, \mathbf{r}^{\prime}\right)=J_{0}\left(\mathbf{r}, \mathbf{r}^{\prime}\right)-j Y_{0}\left(\mathbf{r}, \mathbf{r}^{\prime}\right),
$$

where $J_{0}$ and $Y_{0}$ are the Bessel functions of the $1^{\text {st }}$ and $2^{\text {nd }}$ kind and the function represents outwardly propagating radial waves.

The objective of the overall field scattering problem is thus to find a self-consistent field solution of $(3)$ and $(4]^{2}$ for the specified excitation fields, thereby solving for the unknown surface currents $\{\mathbf{J}, \mathbf{K}\}$ on all surfaces along with the scattered fields in both reflection and transmission regions. For example, the total fields in the reflection region of the metasurface is a sum of the incident fields $\left(\psi_{\text {inc. }}\right)$, and all the reflected scattered fields $\left(\psi_{s}^{-}\right)$generated by the unknown currents, $\{\mathbf{J}, \mathbf{K}\}$ on all the surfaces via (4). Similarly, the total fields in transmission are the total scattered fields $\left(\psi_{s}^{+}\right)$ generated by the same unknown currents. The relationship between the average fields, $\left(\psi_{\text {inc. }}+\psi_{s}^{-}+\psi_{s}^{+}\right) / 2$ and their differences across the metasurfaces $\left(\psi_{s}^{+}-\psi_{\text {inc. }}-\psi_{s}^{-}\right)^{3}$, is given by the GSTCs (3). This integral equation based approach coupled with GSTCs, is referred to here as the IE-GSTC field formulation. For numerical computation, these equations are discretized by segmenting the surfaces following Boundary Element Method (BEM) techniques and assembled in a matrix form for further processing [8], [9], [27], [28].

\footnotetext{
${ }^{1}$ We will denote scattered or radiated fields due to the surface currents by the superscript $\mathrm{s}$ and total fields which include both scattered and incident fields by a lack of superscript. Hence, generally $\mathbf{E}=\mathbf{E}^{\mathrm{s}}+\mathbf{E}^{\mathrm{i}}$ where $\mathbf{E}^{\mathrm{i}}$ is the incident field, for example.

${ }^{2}$ If other objects made of PEC, conductors or dielectric are also part of the scattering problem, then appropriate boundary conditions must be used, in addition to the GSTCs for the respective surfaces. See [8], [32].

${ }^{3}$ Both Electric and Magnetic fields, which are coupled by the GSTCs, unlike standard EFIE, MFIE or CFIE formulations.
} 


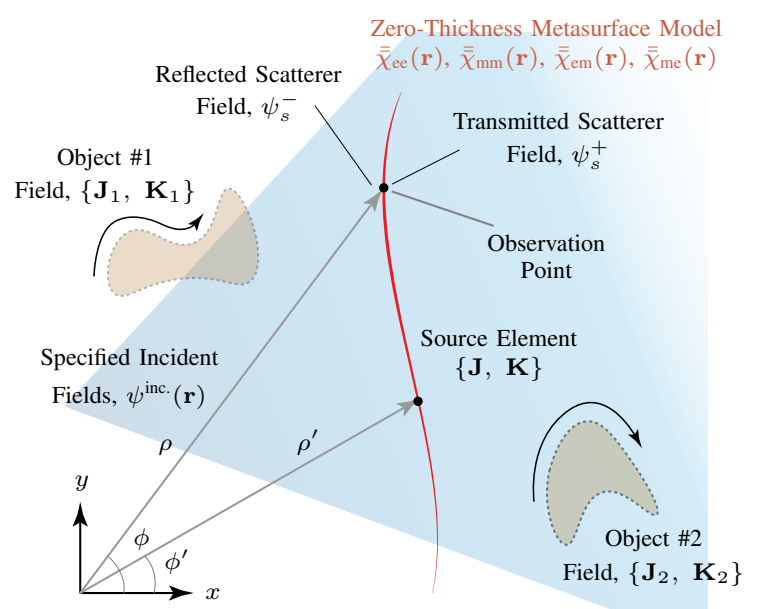

(a)

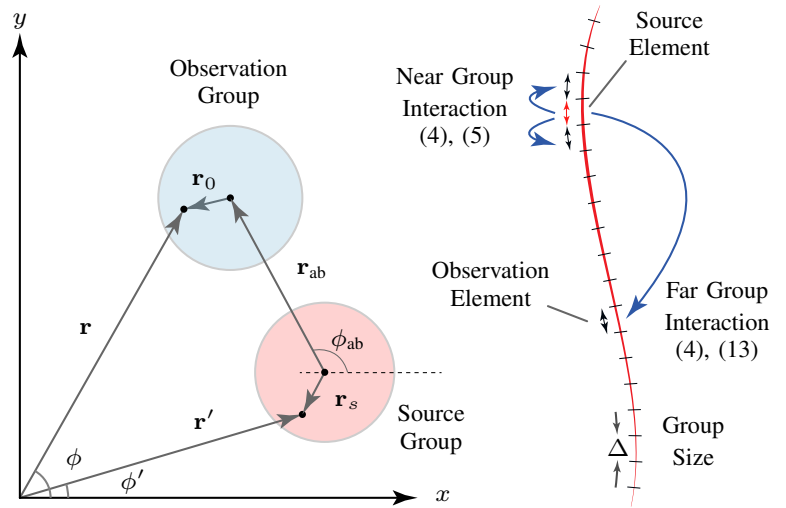

(b)

(c)

Fig. 2. The field scattering problem. a) A zero-thickness metasurface sheet described in terms of their tensorial surface susceptibilities, $\overline{\bar{\chi}}$, which is excited with a given incident fields. b) The general concept of grouping the source and observation elements as a first step in implementing the Fast Multipole Method (FMM). c) The source and observation element grouping applied to a zero thickness metasurface.

\section{Fast Multipole Method (FMM) IE-GSTC}

The IE part of the IE-GSTC formulation involves the matrix solution of a sytems level propagation operator formed from the $\mathcal{L}\{\cdot\}$ and $\mathcal{R}\{\cdot\}$ operators to determine the unknown surface currents. In large computational domains, with a large number of current unknowns direct solution of system matrix will become challenging due to very large memory requirements during solution and the computation time will become prohibitively large An efficient solution to solve this problem and reduce the computation complexity, is based on Fast Multipole Method (FMM) [27]. FMM relies on approximating the Green's function and modeling the electromagnetic effect of a collection of surface currents as if they were replaced by a single element. Consequently, the surface elements are divided into near- and far-groups. While the near-group elements are solved using standard $\mathcal{L}\{\cdot\}$ and $\mathcal{R}\{\cdot\}$ operators, the far group interactions are based on an approximated form of the Green's functions. This formulation is very well suited to the use of an iterative matrix solver as it very efficiently increases the speed of the matrix vector multiplications. The use of a such a solver also removes the very large memory requirements of a direct solver [27].

\section{A. Multipole Expansion of Green's Function between Groups}

For far-group computations (i.e. $r \geq r^{\prime}$ or $\rho>\rho^{\prime}$ ), the Green's function of 6), (2D in our case) can be represented using a series expansion. This allows for the decoupling of the source $\left(\mathbf{r}^{\prime}\right)$ and the observation terms (r). Using the Addition theorem for the Hankel function of the second kind, the 2D electromagnetic Green's function can be written as [40]:

$$
G\left(\mathbf{r}, \mathbf{r}^{\prime}\right)=-\frac{j}{4} \sum_{n=-\infty}^{\infty} J_{n}\left(k r^{\prime}\right) H_{n}^{(2)}(k r) e^{j n\left(\phi-\phi^{\prime}\right)}
$$

To further prepare the Green's function for the far-group calculations, the Bessel functions, $J_{n}\left(k r^{\prime}\right)$ can be rewritten using their integral form as follows

$$
\begin{aligned}
J_{n}\left(k r^{\prime}\right) & =\frac{1}{2 \pi} \int_{0}^{2 \pi} e^{j k r^{\prime} \cos \left(\theta-\phi^{\prime}\right)} e^{-j n\left(\theta-\phi^{\prime}+\frac{\pi}{2}\right)} d \theta \\
& =\frac{1}{2 \pi} \int_{0}^{2 \pi} e^{j \mathbf{k} \cdot \mathbf{r}^{\prime}} e^{-j n\left(\theta-\phi^{\prime}+\frac{\pi}{2}\right)} d \theta,
\end{aligned}
$$

where $\theta$ is an integration variable and $\mathbf{k}$ is a vector with a magnitude equal to the wave number, pointing in the $\theta$ direction, i.e. $\mathbf{k}=k\{\cos \theta \hat{\mathbf{x}}+\sin \theta \hat{\mathbf{y}}\}$. Substituting (8) into (7) and rearranging yields the following expression for the Green's function:

$$
G\left(\mathbf{r}, \mathbf{r}^{\prime}\right) \approx-\frac{j}{8 \pi} \int_{0}^{2 \pi} \sum_{n=-q / 2}^{q / 2} H_{n}^{(2)}(k r) e^{-j n\left(\theta-\phi+\frac{\pi}{2}\right)} e^{i \mathbf{k} \cdot \mathbf{r}^{\prime}} d \theta
$$

where the infinite series has been truncated so that it can be evaluated numerically.

Next, two element groups can be formed - source and observation groups - utilizing the decoupled form of the Green's function, as illustrated in Fig. 2(b) and thus described in terms of group the geometry. For elements in separated groups we can write the Green's function in terms of the position of the elements within their respective groups, and the vector pointing from the centre of the source group to the centre of the observation group. We begin by noting that (see Fig. 2(b)),

$$
\mathbf{r}-\mathbf{r}^{\prime}=\mathbf{r}_{a b}-\left(\mathbf{r}_{s}-\mathbf{r}_{0}\right) .
$$

As the original from of the Green's function is dependent only on the difference between the source and observation vectors, we can write using (10),

$$
G\left(\mathbf{r}, \mathbf{r}^{\prime}\right)=G\left(\mathbf{r}_{a b}, \mathbf{r}_{s}-\mathbf{r}_{0}\right)
$$

Therefore, we can rewrite equation (9), by replacing $r$ and $\phi$ with $r_{a b}$ and $\phi_{a b}$, and similarly $\mathbf{r}^{\prime}$ with $\left(\mathbf{r}_{s}-\mathbf{r}_{0}\right)$, leading to

$$
\begin{aligned}
& G\left(\mathbf{r}, \mathbf{r}^{\prime}\right)=-\frac{j}{8 \pi} \int_{0}^{2 \pi} \sum_{n=-Q / 2}^{Q / 2} H_{n}^{(2)}\left(k r_{a b}\right) \\
& e^{-j n\left(\theta-\phi_{a b}+\frac{\pi}{2}\right)} e^{j \mathbf{k} \cdot\left(\mathbf{r}_{s}-\mathbf{r}_{0}\right)} d \theta,
\end{aligned}
$$

which now represents the Green's function with respect to the two group centers. 


\section{B. FMM Propagation Operators}

With the new form of the Green's function, (12), for $\mathbf{r}>\mathbf{r}^{\prime}$ the two propagation operators, $\mathcal{L}\{\cdot\}$ and $\mathcal{R}\{\cdot\}$ of (4), can now be reformulated. For instance, for the $\mathcal{L}\{\cdot\}$ operator, we can begin by noting for a $2 \mathrm{D}$ case,

$$
\begin{aligned}
\nabla \nabla \cdot\left[G\left(\mathbf{r}, \mathbf{r}^{\prime}\right) \mathbf{C}\left(\mathbf{r}^{\prime}\right)\right]= & \left(C_{x} \frac{\partial^{2} G}{\partial^{2} x}+C_{y} \frac{\partial^{2} G}{\partial x y}\right) \hat{\mathbf{x}} \\
& +\left(C_{x} \frac{\partial^{2} G}{\partial y x}+C_{y} \frac{\partial^{2} G}{\partial^{2} y}\right) \hat{\mathbf{y}}
\end{aligned}
$$

Using (12) in the above equation, and introducing a new matrix operator, $\mathbf{D}_{\mathcal{L}}$,

$$
\mathbf{D}_{\mathcal{L}}=\left[\begin{array}{ccc}
1-\cos ^{2} \theta & -\cos \theta \sin \theta & 0 \\
-\sin ^{2} \theta & 1-\cos \theta \sin \theta & 0 \\
0 & 0 & 1
\end{array}\right]
$$

we get the new form of the $\mathcal{L}\{\cdot\}$ operator as shown in 13a. Similarly, for the $\mathcal{R}\{\cdot\}$, operator, we note that.

$$
\begin{aligned}
\nabla \times\left[G\left(\mathbf{r}, \mathbf{r}^{\prime}\right) \mathbf{C}\left(\mathbf{r}^{\prime}\right)\right]= & \left(C_{z} \frac{\partial G}{\partial y} \hat{\mathbf{x}}-C_{z} \frac{\partial G}{\partial x} \hat{\mathbf{x}}\right) \\
& +\left(C_{y} \frac{\partial G}{\partial x}-C_{x} \frac{\partial G}{\partial y}\right) \hat{\mathbf{x}}
\end{aligned}
$$

Using (12) in the above equation, and introducing a new matrix operator, $\mathbf{D}_{\mathcal{R}}$,

$$
\mathbf{D}_{\mathcal{R}}=\left[\begin{array}{ccc}
0 & 0 & -j k \sin \theta \\
0 & 0 & j k \cos \theta \\
j k \sin \theta & -j k \cos \theta & 0
\end{array}\right],
$$

we get the new form of the $\mathcal{L}\{\cdot\}$ operator as shown in $113 \mathrm{~b}$.

These two operators of (13), due to their decoupled source and observation terms, and the form relative to their group centers, may be expressed in a more compact form as:

$$
\begin{aligned}
(\mathcal{L} \mathbf{C})(\mathbf{r}) & =-\frac{j}{8 \pi} \int_{0}^{2 \pi} T_{s}^{\mathcal{L}}\left(\theta, \mathbf{r}_{s}, \mathbf{C}\right) T_{a b}\left(\theta, \mathbf{r}_{a b}\right) R_{0}\left(\theta, \mathbf{r}_{0}\right) d \theta \\
(\mathcal{R C})(\mathbf{r}) & =-\frac{j}{8 \pi} \int_{0}^{2 \pi} T_{s}^{\mathcal{R}}\left(\theta, \mathbf{r}_{s}, \mathbf{C}\right) T_{a b}\left(\theta, \mathbf{r}_{a b}\right) R_{0}\left(\theta, \mathbf{r}_{0}\right) d \theta
\end{aligned}
$$

in terms of Radiation function, $T_{s}\left(\theta, \mathbf{r}_{s}\right)$ which describes the radiation from the source element to the center of the source group, the Transfer Function, $T_{a b}\left(\theta, \mathbf{r}_{a b}\right)$ which describes the radiation between the source and observation centers, and the Receive Function, $R_{0}\left(\theta, \mathbf{r}_{0}\right)$ which describes receiving the radiation from the observation centers to the observation points. The only difference between the two operators is in the way the source element's radiation contributes to the source centers, via the $\mathbf{D}_{\mathcal{L}}$ and $\mathbf{D}_{\mathcal{R}}$ matrix operators.

This now allows for a very intuitive and convenient interpretation of (14). Consider a single element of the $\mathcal{L}\{\mathbf{C}\}$ or $\mathcal{R}\{\mathbf{C}\}$,

$$
\begin{aligned}
c_{m} & =\frac{-j}{8 \pi} \int_{0}^{2 \pi} R_{0}\left(\theta, \mathbf{r}_{0}\right) \sum_{b} T_{a b}\left(\theta, r_{a b}\right) \sum_{s \in b} T_{s}^{\mathcal{L}, \mathcal{R}}\left(\theta, \mathbf{r}_{s}, \mathbf{C}\right) d \theta \\
& \approx \frac{-j}{8 \pi} \sum_{p=1}^{k} w_{p} R_{0}\left(\theta_{p}, \mathbf{r}_{0}\right) \sum_{b} T_{a b}\left(\theta_{p}, r_{a b}\right) \sum_{s \in b} T_{s}^{\mathcal{L}, \mathcal{R}}\left(\theta_{p}, \mathbf{r}_{s}, \mathbf{C}\right)
\end{aligned}
$$

where $b$ represents a single source group, and $s$, as all the sources in that group. $w_{p}$ is the weighting applied to the sample $\theta_{p}$, witch is determined based on the chosen numerical integration method. In this case, trapezoidal integration was found to give good results when computing (15). All the source element contributions are first aggregated to the source centers via $T^{s}\left(\theta, \mathbf{r}_{s}\right)$. This forms an effective single source. This is next translated to the center of the observation group via $T_{a b}\left(\theta, r_{a b}\right)$, and finally disaggregated to the desired observation points via $R_{0}\left(\theta, \mathbf{r}_{0}\right)$. Compared to the brute force computation of (4), this three-step procedure is significantly more computationally efficient, as transfer, receive and part of radiation function can be precomputed for all values of $\theta$, and stored for repeated usage. This leads to compute time of the order of $O\left(N^{3 / 2}\right)$ compared to $O\left(N^{3}\right)$ when (4) is directly computed [27].

This methodology can be now be easily applied to the zero thickness metasurface where source and observation elements are defined on the surfaces, as illustrated in Fig. 2(c). The number of groups $N_{g}$ needs to be defined and is generally chosen as $N_{g}=\sqrt{N}$, where $N$ is the total number of meshed surface elements on the metasurface [33]. Field contributions next to the source group are modeled using rigorous $\mathcal{L}\{\mathbf{C}\}$ or $\mathcal{R}\{\mathbf{C}\}$ operators, while the rest of the observation elements follow (13).

\section{NumERICAL DEMONSTRATION}

\section{A. Standard IE-GSTC vs FMM IE-GSTC}

In order to validate the implemented FMM algorithm as well as compare its performance with a standard BEM solver, a simple simulation consisting of a finite-sized uniform metasurface of length $\ell$ is set up, as shown in Fig. 3.a). The surfaces are meshed at a density of 10 divisions per wavelength for a total of $N=400$ mesh elements. A Gaussian source of same size, $\ell$, is used to generate a $60 \mathrm{GHz}$ beam to illuminate the surface. The metasurface is designed to achieve a transmission coefficient $T=0.9 j$ and a reflection coefficient $R=0.1$ when illuminated with a plane-wave. The surface susceptibilities can then be synthesized using [18]

$$
\begin{aligned}
\chi_{\mathrm{ee}} & =\frac{2 j}{k}\left(\frac{T+R-1}{T+R+1}\right) \\
\chi_{\mathrm{mm}} & =\frac{2 j}{k}\left(\frac{T-R-1}{T-R+1}\right) .
\end{aligned}
$$

With the surface parameters chosen, now the FMM simulation parameters - number of groups $N_{g}$, number of terms taken in series expansion of Green's function $q$ in (9), number of points, $k$, used to numerically integrate with respect to $\theta$ in (15) - must be selected so that the FMM algorithm runs at an optimal speed, and converges towards the rigorous BEM result.

As stated previously the number of groups $N_{g}$ is typically chosen to be approximately equal to the square root of the number of mesh elements $N$. This maximizes the speed of ) the far field matrix vector multiplication ensuring a compute time which varies as $O\left(N^{3 / 2}\right)$ [33]. In our example, the mesh number of elements is 400 , so the we set $N_{g}=20$. 


$$
\begin{aligned}
& (\mathcal{L C})(\mathbf{r})=-\frac{j}{8 \pi} \int_{0}^{2 \pi} \overbrace{\left[\int_{\ell} e^{j \mathbf{k} \cdot \mathbf{r}_{s}} \mathbf{D}_{\mathcal{L}} \mathbf{C}\left(\mathbf{r}^{\prime}\right) d \mathbf{r}^{\prime}\right]}^{T_{s}^{\mathcal{L}}\left(\theta, \mathbf{r}_{s}, \mathbf{C}\right)} \overbrace{\sum_{n=-q / 2}^{q / 2} H_{n}^{(2)}\left(k \mathbf{r}_{a b}\right) e^{-j n\left(\theta-\phi_{a b}+\frac{\pi}{2}\right)}}^{T_{e^{-j}(\theta) \cdot \mathbf{r}_{0}}^{R_{0}\left(\theta, \mathbf{r}_{0}\right)}} d \theta \\
& (\mathcal{R} \mathbf{C})(\mathbf{r})=-\frac{j}{8 \pi} \int_{0}^{2 \pi} \underbrace{\left[\int_{\ell} e^{j \mathbf{k} \cdot \mathbf{r}_{s}} \mathbf{D}_{\mathcal{R}} \mathbf{C}\left(\mathbf{r}^{\prime}\right) d \mathbf{r}^{\prime}\right]}_{T_{s}^{\mathcal{R}}\left(\theta, \mathbf{r}_{s}, \mathbf{C}\right)} \underbrace{\sum_{n=-q / 2}^{q / 2} H_{n}^{(2)}\left(k \mathbf{r}_{a b}\right) e^{-j n\left(\theta-\phi_{a b}+\frac{\pi}{2}\right)}}_{T_{a b}\left(\theta, \mathbf{r}_{a b}\right)} \underbrace{e^{-j \mathbf{k} \cdot \mathbf{r}_{0}}}_{R_{0}\left(\theta, \mathbf{r}_{0}\right)} d \theta
\end{aligned}
$$

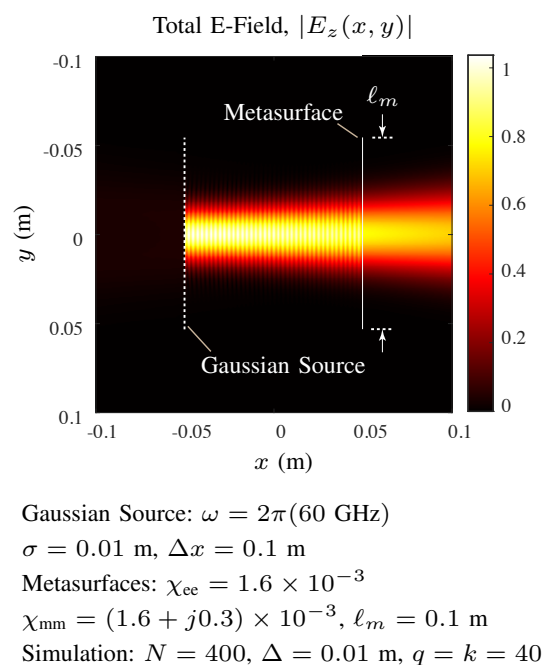

(a)
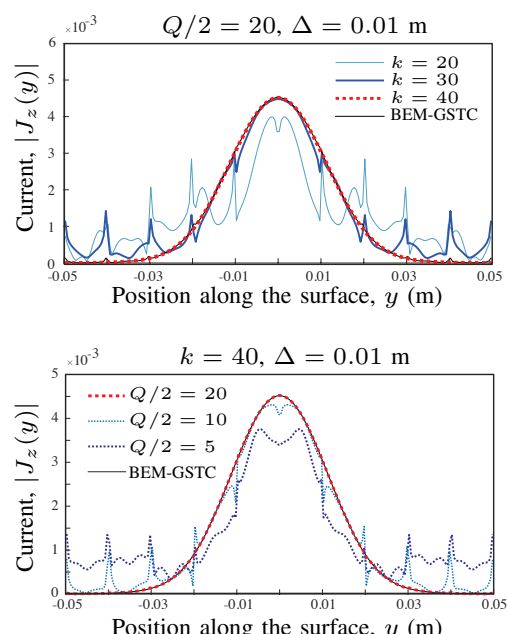

(b)

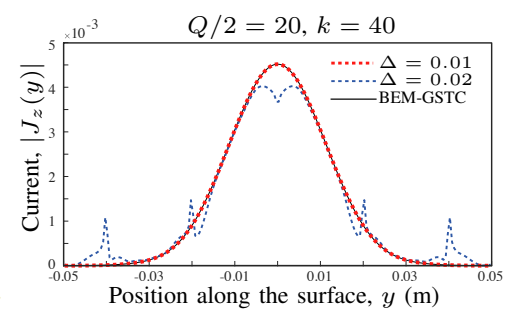

(c)

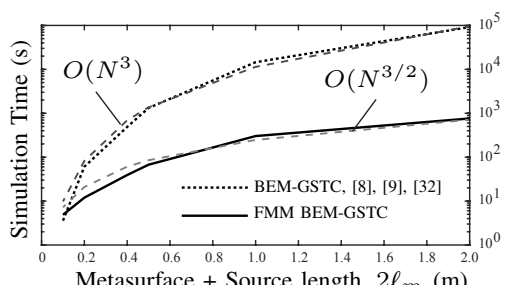

(d)

Fig. 3. Comparison between the proposed FMM IE-GSTC with the standard IE-GSTC formulation for a finite-sized uniform metasurface. a) 2D Total E-fields when the surface is normally illuminated by a Gaussian beam. b) Effect of the series truncation in (9) (bottom) and number of integration points in (15) (top) on the surface current computation for a fixed group size, $\Delta$. c) Effect of the group size $\Delta$ on the surface currents. d) Comparison between the computation time between FMM IE-GSTC and the standard IE-GSTC for varying surface length, $\ell_{m}$.

Furthermore, the following empirical formula can be used to determine when to truncate the series expansion of the Green's function [33]

$$
\frac{q}{2}=k D+5 \ln (k D+\pi)
$$

Where $\mathrm{D}$ is the diameter of the groups $(D=\Delta \sqrt{2})$. In general, $q=2 N_{e}$, where $N_{e}$ is the number of elements per group, is enough to ensure convergence and is often smaller than what is given by (17). For this particular setup we have $N=400$ elements divided into $N_{g}=20$ groups, resulting in $N_{e}=$ 20 elements per group. Therefore the series expansion of the Green's function is truncated at $q=40$. For the numerical integration with respect to $\theta$, we see from (13) that if we were to represent this function using discrete values of $\theta$ we would need $q$ samples in order to satisfy the Nyquist sampling theorem. For our chosen value of $q=40$, we therefore set $k=40$.

Next, the iterative solver, and its parameters are to be chosen. MATLAB has several iterative matrix solvers built in. In general GMRES can be applied to most problems, but in some cases, other methods such as the stabilized biconjugate gradient method and conjugate gradient squared method may be able solve the system more efficiently although their convergence is less predictable. For this example the stabilized bi-conjugate gradient method was used as it was able to converge faster than GMRES [41]. The tolerance of the solver was set to $10^{-6}$ Additionally, a set of preconditioner matrices can be chosen into improve the conditioning of the system and speed up the convergence of the iterative solver. For this example ILU(0) preconditioning was used with a drop tolerance of $10^{-6}$.

With the initial FMM parameters set, the convergence of the accelerated FMM IE-GSTC solver can now be tested against a standard BEM-GSTC solver. To do this, the FMM parameters are set to their standard values given above, and we vary $q, k$ and the number of groups, $N_{g}$. For various cases, the electric current density, $\mathbf{J}(y)$ on the metasurface is calculated and compared to the standard BEM result. Fig. 3. (b) shows the effect of $q$ and $k$ on $\mathbf{J}$, when the group size $N_{g}$ is fixed. From these results it is clear that the FMM results converge to the BEM-GSTC results when $q=40$ and $k=40$, as expected, but if either of these values are lowered, the FMM no longer produces accurate results.

Next, the effect of group size on convergence can be examined, and is shown in Fig. 3(c). We find that when the group size $\Delta=2 \ell / \sqrt{N}=0.01 \mathrm{~m}, \quad(20$ groups in 
total) the FMM converges to the BEM-GSTC result. However, when we increase the group size to $0.02 \mathrm{~m}$, for instance, result significantly deviates. This is because as the group size grows, the series expansion truncation, $q$, and by extension, the number of numerical integration points, $k$, have to increase accordingly. Since these values were held constant, the far group representation of the Green's function was no longer valid.

Now that the FMM results have been verified, the scalability of the FMM can be compared to BEM-GSTC. This is done by increasing the length, $2 \ell_{m}$ of the two surfaces (source and the metasurfaces) in Fig. 3 (a) and comparing the time it takes both methods to solve for the current densities under converged conditions. These results are shown in Fig. 3(d). The results show that for smaller lengths, the BEM outperforms the FMM in terms of speed, but as the length of the surfaces is increased, the FMM solves the system of equations significantly faster with a difference of over two orders of magnitude, when the surfaces are $2 \mathrm{~m}$ long. This very closely follows the expected simulation time of order $O\left(N^{3}\right)$ and $O\left(N^{3 / 2}\right)$ for BEM-GSTC and FMM BEM-GSTC solvers, respectively. From these results it is clear that in order to obtain full wave solutions for metasurfaces placed in electrically large simulations, the FMM is very effective method to accelerate the standard BEMGSTCs solver.

\section{B. Complex Indoor environment}

Let us apply the FMM BMM-GSTC to a practical scenario where the scattered fields need to be computed in a large computational domain i.e., wireless communication. Fig. 4 shows an example of an arbitrarily chosen two room scenario for illustration, where each of the room consists of doors and windows, which are connected to each other by a window in the partition wall. While the doors and windows are modeled as transparent surfaces, walls are made up of typical dielectric material $\left(20 \mathrm{~cm}\right.$ thick plasterboard with $\epsilon_{r}=2.02$ and $\tan \delta=0$, for example [42]). A fixed source is located along one of the walls in room $\# 1$, which is radiating a fixed beam at a chosen operation frequency (e.g. $6 \mathrm{GHz}$ for microwave communication). Our first goal is obtain the field coverage inside this reference environment.

In order to make some simplifications to this overall large problem, and considering we are interested in fields inside the rooms only, we create an approximate equivalent model of the dielectric walls, by replacing them with a zero thickness surface susceptibility model in terms of $\chi_{\mathrm{ee}}$ and $\chi_{\mathrm{mm}}$. This is achieved by using the Fresnel transmission and reflection coefficients of a dielectric slab and using them in 16 to compute the two tangential susceptibilities. Windows and doors are assumed to be perfectly transparent and are modeled by setting all susceptibilities to zero. It should be noted that these equivalent surface susceptibilities are strictly valid for normal plane-wave incidence only, and a more sophisticated tensorial models must be used to properly account for angular scatterings.

Next, the total fields are computed using the FMM BEMGSTC solver assuming a Gaussian beam generated by the

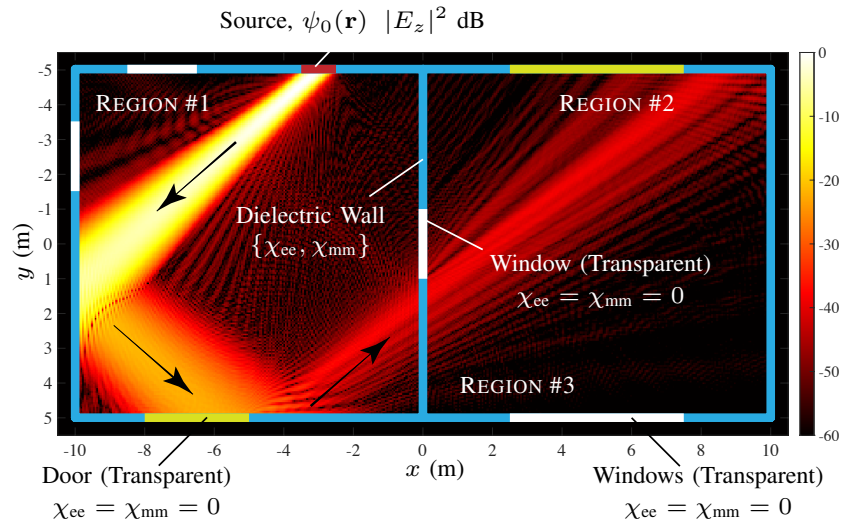

Fig. 4. Illustrative example of a wireless environment two large connected rooms with various windows and doors where a fixed signal source is placed, showing its typical coverage at $6 \mathrm{GHz}$. The various simulation parameters are: $\omega=2 \pi(6 \mathrm{GHz})$, Source $\sigma_{\psi}=0.2 \mathrm{~m}, \theta_{\psi}=138.24^{\circ}$, Wall: $\chi_{\mathrm{ee}}=$ $-0.0299+0.0074 j, \chi_{\mathrm{mm}}=-0.0491-0.0122 j$.

source. Fig. 4 shows the field distribution in the entire computation region. The beam propagates with passive reflections off various walls, windows and doors, eventually resulting in particularly a weak signal in room \#2 and limited signal coverage overall. Let us next consider that we are particularly interested in enhancing this signal coverage in three specific regions (marked region \#1 - \#3 in Fig. 4). To achieve this goal, we will now design and strategically install metasurfaces in specific locations within the environment.

Among multiple potential solutions to design the wireless environment using metasurfaces, one example plan is shown in Fig. 5(a). We install three metasurfaces (marked MS\#1, \#2 and \#3), one on each of the walls opposite to the source and one on the connecting window between the two rooms. The first metasurface \#1 takes the incident source field and reflects a Gaussian beam of smaller width towards the second metasurface, thus acting as a good reflector with steerable beam following a Generalized Snell's law. The second metasurface \#2 is a reflective beam splitter that generates two gaussian beams, one illuminating the upper left hand corner of the first room targeting region \#1, and the other illuminating the metasurface on the adjoining wall. The third metasurface \#3 is an active transmissive beam splitter with gain designed to generate two beams inside Room \#2, targeting the desired regions \#1 and \#2.

Next, to follow this plan, metasurfaces are synthesized assuming the incident fields are Gaussian beams for simplicity and can be described by

$$
E_{z}=\frac{\sigma_{0}}{\sigma} \exp \left\{-\frac{x_{s}^{2}}{2 \sigma^{2}}\right\} e^{j k r \cos \theta},
$$

where $x_{s}$ is the position along the metasurface, $r$ is the distance between the source of the beam and the surface, $\theta$ is the incidence angle, $\sigma$ is the beam-width at the surface, and $\sigma_{0}$ is the initial beam-width. The increase in the Gaussian beam width can be described by standard paraxial wave-propagation as 17

$$
\sigma(z)=\sigma_{0} \sqrt{1+\left(\frac{r}{z_{r}}\right)^{2}}, \text { with } z_{r}=\frac{2 \pi \sigma_{0}^{2}}{\lambda}
$$




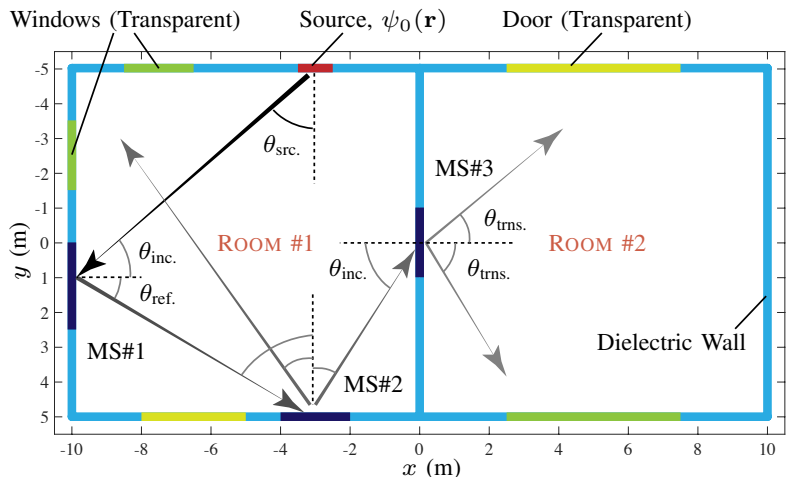

(a)

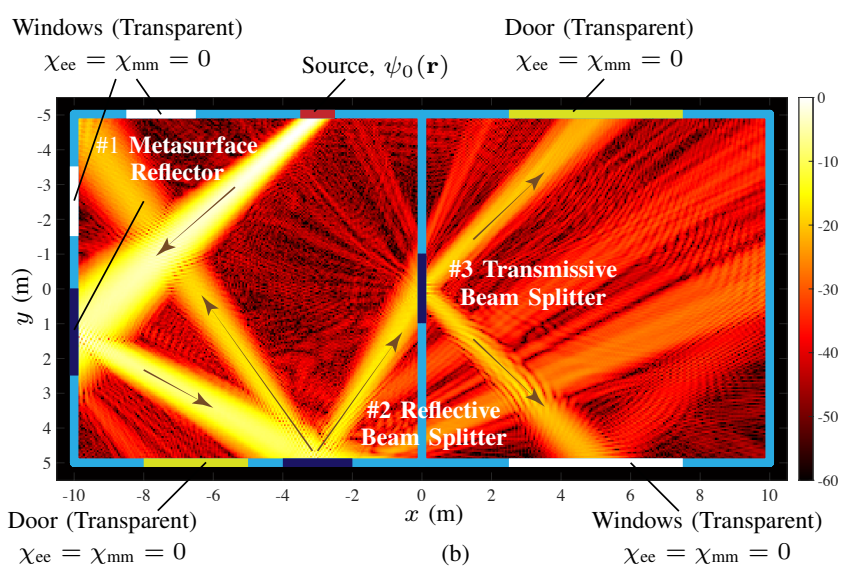

Fig. 5. Possible plan to strategically place engineered metasurfaces to route the electromagnetic waves to desired locations, thereby significantly increasing signal coverage. Computed total fields, abs $\{|E|\}$ shown for the two cases of a) no metasurfaces (base case), and b) with three specific metasurfaces. The various metasurface design parameters are provided in Tab. I. MS\#1 is $2.5 \mathrm{~m}$ long where as MS\#2 and MS\#3 are $2 \mathrm{~m}$ long.

TABLE I

PARAmeters used For Metasurface Synthesis OF Fig. 5 $\left(\sigma_{\mathrm{REF} .}=\sigma_{\mathrm{TRNS} .}=0.2 \mathrm{M}\right.$ IN ALL CASES $)$

\begin{tabular}{c|ccccc} 
& $\theta_{\text {inc. }}$ & $\sigma_{\text {inc. }}$ & $\theta_{\text {trns. }}$ & $\theta_{\text {ref. }}$ & Gain \\
\hline Reflector \#1 & $42^{\circ}$ & $0.42 \mathrm{~m}$ & - & $28^{\circ}$ & 1 \\
Splitter \#2 & $62^{\circ}$ & $0.37 \mathrm{~m}$ & - & $\left\{35^{\circ}, 31^{\circ}\right\}$ & 1 \\
Splitter \#3 & $59^{\circ}$ & $0.31 \mathrm{~m}$ & $\left\{45^{\circ}, 45^{\circ}\right\}$ & - & 2 \\
\hline
\end{tabular}

With these equations, an approximate expression can be found for the incident field on the metasurface based on either the Gaussian source, or the specified scattered fields from the previous metasurface. The scattered fields on the metasurface are then specified in order to achieve the surface's desired functionality. With these approximate expressions for the incident, reflected and transmitted fields known, the desired surface susceptibilities can be calculated using

$$
\begin{aligned}
\chi_{\mathrm{ee}} & =\frac{-\Delta H_{\|}}{j \omega \epsilon E_{z, a v}} \\
\chi_{\mathrm{mm}} & =\frac{-\Delta E_{z}}{j \omega \mu H_{\|, a v}} .
\end{aligned}
$$

and are summarized in Tab I.

With the surface conditions set, the simulation parameters can now be determined. The FMM parameters $q, N_{q}, k$ are set using the same criteria discussed in the previous section. Now the iterative solver parameters are chosen. Due to the size of this environment, convergence was an issue. For this reason, GMRES was the chosen iterative algorithm as it is the most reliable. As previously, ILU(0) preconditioning was used. This time, the drop tolerance was increased to $10^{-4}$ to speed up the generation of the preconditioner. To further improve the convergence, MATLAB's built in equilibrate function was used to permute and scale the system of equations to reduce the condition number of the system and lead to a more efficient iterative solution. Finally, the tolerance of the iterative solver was set to $10^{-5}$ for acceptable convergence.

The total fields are next computed inside the two rooms in the presence of the three metasurfaces, and the resulting field distribution is shown in Fig. 5. (b). A clear and a significant improvement in the signal coverage is observed across the two rooms, in comparison to the reference case of Fig. 4, with the desired functionality of the three metasurfaces successfully achieved, as apparent from their respective scattered fields. Moreover, as desired and expected, the signal in the three targeted regions has been boosted, thanks to the strategic routing through the three metasurfaces. While this example illustrates leveraging the wave transformation capabilities of metasurfaces to control wave propagation inside complex environments, it clearly shows the importance and applicability of the proposed FMM IE-GSTC solver in computationally large problems, that a standard IE-GSTC solver may not be able to handle.

\section{CONCLUSION}

An accelerated IE-GSTC solver for determining the scattered fields from an electrically large electromagnetic metasurface based on FMM has been proposed and numerically demonstrated in 2D. The proposed method builds upon the standard IE-GSTC simulation framework, where a general metasurface is expressed using an equivalent zero thickness sheet model described using surface susceptibilities and the fields around it satisfy the GSTCs. The field propagation is implemented using an IE formulation. It has been shown that FMM accelerates the computation speed by dividing the unknown current elements on the metasurface into near- and far-groups, where either the rigorous or the approximated Green's functions is used, respectively. Using numerical example, the speed improvement of the FMM IE-GSTC method $\left\{O\left(N^{3 / 2}\right)\right\}$ over the standard IE-GSTC $\left\{O\left(N^{3}\right)\right\}$ method has been confirmed. Finally, usefulness of the FMM IEGSTC has been demonstrated by applying it to solve electromagnetic wave propagation inside an electrically large radio environment equipped with strategically placed metasurfaces to improve signal coverage in blind areas. Future efforts in this direction may involve subsequent improvements in the FMM implementation based on various known techniques such as Fast Fourier Transforms (FFTs) and multilevel FMM, for instance [43]-[45] and extension to 3D. While a single application case of the radio environment has been used here for demonstration, the proposed FMM IE-GSTC is expected 
to be useful in variety of other electrically large simulation problems to achieve an optimal use of available computational resources and reduce simulation times.

\section{ACKNOWLEDGEMENTS}

The authors acknowledge funding from the Department of National Defence's Innovation for Defence Excellence and Security (IDEaS) Program in support of this work.

\section{REFERENCES}

[1] C. L. Holloway, E. F. Kuester, J. A. Gordon, J. O'Hara, J. Booth, and D. R. Smith, "An overview of the theory and applications of metasurfaces: The two-dimensional equivalents of metamaterials," IEEE Antennas Propag. Mag., vol. 54, no. 2, pp. 10-35, 2012.

[2] H.-T. Chen, A. J. Taylor, and N. Yu, "A review of metasurfaces: physics and applications," Rep. Prog. Phys., vol. 79, no. 7, p. 076401, 2016.

[3] H.-H. Hsiao, C. H. Chu, and D. P. Tsai, "Fundamentals and applications of metasurfaces," Small Methods, vol. 1, no. 4, p. 1600064, 2017.

[4] X. Luo, "Principles of electromagnetic waves in metasurfaces," Sci. China Phys. Mech. Astron., vol. 58, no. 9, p. 594201, 2015.

[5] S. B. Glybovski, S. A. Tretyakov, P. A. Belov, Y. S. Kivshar, and C. R Simovski, "Metasurfaces: From microwaves to visible," Phys. Rep., vol 634, pp. 1-72, 2016

[6] A. Arbabi and A. Faraon, "Fundamental limits of ultrathin metasurfaces," Sci. Rep., vol. 7, p. 43722, 2017.

[7] S. S. Bukhari, J. Y. Vardaxoglou, and W. Whittow, "A metasurfaces review: Definitions and applications," Appl. Sci., vol. 9, no. 13, p. 2727, 2019.

[8] T. J. Smy and S. Gupta, "Surface susceptibility synthesis of metasurface skins/holograms for electromagnetic camouflage/illusions," IEEE Access, vol. 8, pp. 226866-226886, Dec 2020.

[9] T. J. Smy, S. A. Stewart, and S. Gupta, "Surface susceptibility synthesis of metasurface holograms for creating electromagnetic illusions," IEEE Access, vol. 8, pp. 93 408-93 425, 2020.

[10] M. D. Renzo, M. Debbah, D.-T. Phan-Huy, A. Zappone, M.-S Alouini, C. Yuen, V. Sciancalepore, G. C. Alexandropoulos, J. Hoydis, H. Gacanin, J. de Rosny, A. Bounceu, G. Lerosey, and M. Fink, "Smart radio environments empowered by ai reconfigurable meta-surfaces: An idea whose time has come," arXiv:1903.08925. [Online]. Available: https://arxiv.org/abs/1903.08925, Mar 2019.

[11] Q. Wu and R. Zhang, "Towards smart and reconfigurable environment: Intelligent reflecting surface aided wireless network," arXiv:1905.00152. [Online]. Available: https://arxiv.org/abs/1905.00152, Apr 2019.

[12] E. Basar, "Reconfigurable intelligent surface-based index modulation: A new beyond mimo paradigm for 6g," arXiv:1904.06704. [Online]. Available: https://arxiv.org/abs/1904.06704, Apr 2019.

[13] S. Stewart, Y. L. de Jong, T. J. Smy, and S. Gupta, "Ray-optical evaluation of scattering from electrically large metasurfaces characterized by locally periodic surface susceptibilities," arXiv preprint arXiv:2102.07041, 2021

[14] M. Albani, G. Carluccio, and P. H. Pathak, "Uniform ray description for the po scattering by vertices in curved surface with curvilinear edges and relatively general boundary conditions," IEEE Transactions on Antennas and Propagation, vol. 59, no. 5, p. 1587-1596, 2011.

[15] G. Carluccio, M. Albani, and P. Pathak, "Uniform asymptotic evaluation of surface integrals with polygonal integration domains in terms of utd transition functions," IEEE Transactions on Antennas and Propagation, vol. 58, no. 4, p. 1155-1163, 2010.

[16] Y. L. C. de Jong, "Uniform ray description of physical optics scattering by finite locally periodic metasurfaces," submitted to IEEE Trans. Antennas Propagat., 2021.

[17] J. Goodman, Introduction to Fourier Optics. Roberts and Company Publishers; 3rd Edition edition, 2004.

[18] K. Achouri, M. A. Salem, and C. Caloz, "General metasurface synthesis based on susceptibility tensors," IEEE Trans. Antennas Propag., vol. 63 no. 7, p. 2977-2991, Jul 2015

[19] X. Liu, F. Yang, M. Li, and S. Xu, "Generalized boundary conditions in surface electromagnetics: Fundamental theorems and surface characterizations," Applied Sciences, vol. 9, no. 9, 2019.

[21] Y. Vahabzadeh, N. Chamanara, K. Achouri, and C. Caloz, "Computational analysis of metasurfaces,", IEEE J. Multiscale Multiphys. Comput. Tech., vol. 3, pp. 37-49, 2018.

[20] _ - "Generalized boundary conditions in surface electromagnetics: Fundamental theorems and surface characterizations," Appl. Sci., vol. 9, no. 9,2019
[22] Y. Vahabzadeh, K. Achouri, and C. Caloz, "Simulation of metasurfaces in finite difference techniques," IEEE Trans. Antennas Propag., vol. 64, no. 11, p. 4753-4759, Nov. 2016.

[23] Y. Vahabzadeh, N. Chamanara, and C. Caloz, "Generalized sheet transition condition fdtd simulation of metasurface," IEEE Trans. Antennas Propag., vol. 66, no. 1, pp. 271-280, Jan. 2018

[24] S. Sandeep, J.-M. Jin, and C. Caloz, "Finite-element modeling of metasurfaces with generalized sheet transition conditions," IEEE Trans. Antennas Propag., vol. 65, no. 5, p. 2413-2420, May 2017.

[25] N. Chamanara, K. Achouri, and C. Caloz, "Efficient analysis of metasurfaces in terms of spectral-domain gstc integral equations,", IEEE Trans. Antennas Propag., vol. 65, no. 10, p. 5340-5347, Oct 2017.

[26] S. A. Stewart, T. J. Smy, and S. Gupta, "Finite-difference time-domain modeling of space-time-modulated metasurfaces," IEEE Trans. Antennas Propag., vol. 66, no. 1, pp. 281-292, Jan 2018.

[27] W. C. Gibson, The Method of Moments in Electromagnetics. London, U.K.: Chapman \& Hall, 2008.

[28] W. Chew, M. Tong, and B. Hu, Integral Equation Methods for Electromagnetic and Elastic Waves. San Rafael, CA, USA: Morgan \& Claypool Publishers, 2009.

[29] S. Kagami and I. Fukai, "Application of boundary-element method to electromagnetic field problems (short papers)," IEEE Trans. Microw. Theory Techn., vol. 32, no. 4, p. 455-461, Apr. 1984.

[30] S. A. Stewart, S. Moslemi-Tabrizi, T. J. Smy, and S. Gupta, "Scattering field solutions of metasurfaces based on the boundary element method for interconnected regions in 2-d," IEEE Trans. Antennas Propag., vol. 67 , no. 12 , p. 7487-7495, Dec 2019.

[31] V. Tiukuvaara, T. Smy, and S. Gupta, "Floquet analysis of space-time modulated metasurfaces with lorentz dispersion," IEEE Trans. Antennas Propag., 2021

[32] T. J. Smy, V. Tiukuvaara, and S. Gupta, "IE-GSTC metasurface field solver using surface susceptibility tensors with normal polarizabilities," 2021.

[33] S. S. Bindiganavale and J. L. Volakis, "Comparison of three fmm techniques for solving hybrid fe-bi systems," IEEE Trans. Antennas Propag., vol. 39, no. 4, p. 47-60, Aug 1997.

[34] R. Coifman, V. Rokhlin, and S. Wandzuraz, "The fast multipole method for the wave equation: A pedestrian prescription," IEEE Trans. Antennas Propag., vol. 35, no. 3, p. 7-12, Jun 1993.

[35] D. O. Almeida, R. L. S. Adriano, R. C. Mesquita, and J. A. Vasconcelos, "The fast multipole method for $2 \mathrm{~d}$ wave equation solved by the hybrid finite element-boundary element technique," Journal of Microwaves and Optoelectronics, vol. 3, no. 3, p. 54-57, Dec 2003.

[36] I. Kavanagh, V. Pham-Xuan, M. Condon, and C. Brennan, "A method of moments based indoor propagation model," in 2015 9th European Conference on Antennas and Propagation (EuCAP), Apr. 2015, pp. 1-

[37] I. Kavanagh and C. Brennan, "A hybrid $2 \mathrm{~d}$ to $3 \mathrm{~d}$ full wave indoor propagation model," in 2016 International Conference on Electromagnetics in Advanced Applications (ICEAA), Sept. 2016, pp. 748-751.

[38] — "Preliminary investigation of power delay profile computation from full wave frequency domain indoor propagation model," in 2017 11th European Conference on Antennas and Propagation (EUCAP), Mar. 2017, pp. 2242-2245.

[39] A. Lozano and N. Jindal, "Transmit diversity vs. spatial multiplexing in modern mimo systems," Trans. Wireless. Comm., vol. 9, no. 1, pp. 186-197, Jan. 2010

[40] A. C. Balanis, Advanced Engineering Electromagnetics. Hoboken,NJ, USA: Wiley, 1989

[41] Y. Saad, Iterative Methods for Sparse Linear Systems. PWS Publishing Company, 1996.

[42] I. Cuinas and M. G. Sanchez, "Permittivity and conductivity measurements of building materials at $5.8 \mathrm{ghz}$ and $41.5 \mathrm{ghz}$," Wireless Personal Communications, vol. 20, pp. 93-100, 2002.

[43] J.-M. Song and W. C. Chew, "Multilevel fast multipole algorithm for solving combined field integral equations of electromagnetic scattering," Microw. Opt. Tech. Lett., vol. 10, no. 1, p. 14-19, Sept. 1995.

[44] J.-M. Song, C. Lu, and W. C. Chew, "Multilevel fast multipole algorithm for electromagnetic scattering by large complex objects," IEEE Trans. Antennas Propag, vol. 45, no. 10, pp. 1488-1493, Oct. 1997.

[45] R. L. Wagner, J. Song, and W. C. Chew, “"monte carlo simulation of electromagnetic scattering from two-dimensional random rough surfaces," IEEE Trans. Antennas Propag, vol. 45, no. 2, pp. 235-245, Feb. 1997. 